\title{
28 Research Suare \\ Manufacturing Cellulose Nanofibers Using the Water Jet Method and Their Applications
}

Kota Ogura ( $\nabla$ k.ogura@sugino.com )

Sugino Machine Limited

\section{Research Article}

Keywords: Water Jet, Cellulose Nanofiber, Dispersant, Natural Rubber, Polymer

Posted Date: May 27th, 2021

DOl: https://doi.org/10.21203/rs.3.rs-546068/v1

License: (c) (i) This work is licensed under a Creative Commons Attribution 4.0 International License.

Read Full License 


\section{Abstract}

Sugino Machine Ltd. manufactures and sells equipment for cutting, washing, atomization, and fibrillation of objects using water jet technology. By applying this technology, cellulose nanofibers (CNFs) have been successfully and efficiently produced. The feature of CNFs manufactured by water jet is that the crystallinity and polymerization degree do not decrease. A drying method has also been developed because CNFs are obtained in the aqueous dispersion state. Herein, we present their use as particle dispersant and reinforcing filler for natural rubber (NR) as case studies of CNF aqueous dispersion applications. When CNFs was used as a particle dispersant, good dispersion of water-repellent $\mathrm{TiO}_{2}$, diamond powder and inorganic pigment was achieved. When CNFs was used as a filler for NR, a specific reinforcing effect was realized. As an example of CNF application as dry powders, their use with a resin additive is investigated. When CNF dry powders was used as a filler for resin, a specific reinforcing effect was realized.

\section{Introduction}

A water jet is a narrow, high-speed jet produced by passing ultra-high-pressure water through a nozzle. The energy of the jet can be varied by changing the initial pressure of water, thereby obtaining effects such as "cutting," "washing," "atomizing," and "fibrillating". In other words, various processes can be performed depending on the pressure of water. Wet jet mill equipment based on our proprietary water jet technology is employed in various industries because it can pulverize, disperse, fibrillate, and emulsify at a nanoscopic level.

However, cellulose nanofibers (CNFs) produced by fibrillating pulp have attracted attention worldwide because of their excellent characteristics, such as high strength, low thermal expansion, light weight, and high specific surface area, in addition to being produced from a renewable resource existing in nature. Cellulose fibers in plants are tightly bound to each other through intermolecular hydrogen bonds; thus, it is not easy to break them to produce CNFs. Therefore, a method for efficiently breaking intermolecular hydrogen bonds chemically and/or physically to produce CNFs has been developed and practically used, and a wide variety of CNFs are currently available (Saito et al. 2006; Nakagaito and Yano 2004). The example of manufacturing CNFs by the water jet is also reported (Watanabe et al. 2011; Chaikaew et al. 2012). However, the production of the CNF aqueous dispersions was generally carried out at a low concentration of $2 \mathrm{wt} \%$ or less.

Our company has applied water jet technology to develop equipment capable of efficiently producing CNFs. This system enables the production of CNF aqueous dispersions at high concentrations (approximately $10 \mathrm{wt} \%$ ). CNFs manufactured via fibrillation, also known as the water jet method, are sold under the trade name "BiNFi-s." This paper reports the production of CNFs by the water jet method and their drying method. In addition, examples of applications using the thus-obtained CNFs are also reported. 


\section{Methods}

\subsection{Manufacturing of CNFs by Water Jet Method}

Three types of powdered cellulose with different fiber lengths (ultra-long, standard, and ultra-short) derived from softwood or hardwood were used as cellulose raw materials. The nanofiber manufacturing equipment utilizing the water jet method developed in our company is schematically illustrated in Fig. 1. A cellulose aqueous dispersion was charged into the raw material tank, fed into two intensifiers using a feed pump, and pressurized up to $245 \mathrm{MPa}$. The pressurized dispersion was ejected from two diamond nozzles facing each other in an impingement chamber. The cellulose-containing water jets reached a speed of approximately $700 \mathrm{~m} / \mathrm{s}$ (Mach 2), while colliding with each other in the chamber. Then, fibrillation was driven by (a) shearing force caused by high speed, (b) collision force between jets, and (c) impact force by bursting cavitation bubbles in the impinging jet. Because of the increase in temperature in following this treatment, the cellulose aqueous dispersion was cooled by passing through a heat exchanger and then recovered. CNFs form a three-dimensional network in water, resulting in a uniform and highly viscous fluid. The maximum manufacturable concentration varies depending on the cellulose fiber length, but is generally $10 \mathrm{wt} \%$. The characteristics of this method are described below.

1. Since fibrillation is possible only with water and raw materials, it is friendly to the environment and human body (don't use organic solvent).

2. Contamination of the resulting dispersion is extremely low because no grinding media are used.

3. CNFs can be obtained in a short time and with high efficiency because of the high energy density of the water jet.

4. Continuous processing is possible, and mass production can be easily scaled up by numbering-up of the equipment.

5. Treatment of raw materials with high concentration and viscosity is also possible.

6. Because the fibrillation force is not excessive, it does not break the fiber shape of the raw material.

7. The physical properties of the resulting CNFs can be controlled by controlling the pressure and the number of collisions.

The driving force of fibrillation is described as follows:

(a) Shearing force

Shear force increases as the cellulose aqueous dispersion flows at a high speed through a narrow space. Because velocity is proportional to pressure, the higher the pressure, the greater is the shear force.

(b) Collision force

Collision force is proportional to the square of velocity. A high-speed collision is subject to a large collision force. However, because the jet ejected from the nozzle is attenuated at a small distance, it is 
important to impinge the jet while maintaining a fast speed before attenuation.

(c) Impact force

Impact force is generated when the cavitation bubbles disappear.

When high-pressure water is jetted, the surrounding water is subjected to shearing, drops to saturated water vapor pressure, and boils. Bubbles generated by such boiling are called cavitation bubbles, and their disappearance upon return to higher pressures is termed cavitation phenomenon. A cavitation bubble generates a large impulsive force while disappearing, owing to the inflow of a micro-jet at an ultrahigh speed. It is important to efficiently generate and extinguish cavitation bubbles. The impulsive force caused by their disappearance increases with the addition of resistance in the jet channel. However, adding resistance in the jet channel promotes the attenuation of the jet.

In summary, (b) and (c) are in a trade-off relationship. To perform fibrillation efficiently, it is necessary to utilize the three forces in a well-balanced manner. Further, because the optimum conditions depend on the raw material and purpose, the present method is characterized by a channel shape and resistance specific to the fibrillation of cellulose.

\subsection{Basic characterization of CNF water dispersions}

Fiber shapes were observed by field-emission scanning electron microscopy (FE-SEM SU8240, Hitachi High-Tech Corporation, Tokyo, Japan). The degree of polymerization was assessed by measuring the pulp viscosity (relative viscosity method) using a copper(II) ethylenediamine solution. The crystal structure was determined via X-ray diffraction (XRD) analysis (40 kV-20 mA, 2q=2-40 ${ }^{\circ}$ ).

\subsection{Manufacturing of CNF dry powder via the Sugino Machine Limited original method}

CNFs prepared by the water jet method are obtained as aqueous dispersions. Therefore, it is necessary to dry them to further compound them with a hydrophobic resin. For this purpose, we developed an original drying process.

Aqueous dispersions of standard length (BiNFi-s WFo, Sugino Machine Limited, Toyama, Japan) and ultra-short length (BiNFi-s FMa, Sugino Machine Limited, Toyama, Japan) CNFs were used to produce standard (WFo-UNDP) and ultra-short (FMa-UNDP) CNF dry powders. The particle size of the CNF dry powders was evaluated using a laser diffraction/scattering particle size distribution measuring device (LA-300, HORIBA, Ltd., Kyoto, Japan).

\section{Results And Discussion}




\subsection{Basic properties of CNF aqueous dispersions}

The FE-SEM images of CNFs produced by the water jet method from cellulose with different fiber lengths are shown in Fig. 2. The fibrillation conditions were identical for all the samples. Both raw materials present an almost uniform fiber diameter of 10-20 nm; lengths were in the order of $10 \mathrm{~mm}$ or more for the ultra-long CNFs, several $\mathrm{mm}$ for the standard ones and approximately $1 \mathrm{~mm}$ for the ultra-short ones.

The change in the degree of polymerization of standard and ultra-short cellulose after treatment with the water jet method is shown in Fig. 3, while the XRD patterns of standard cellulose are shown in Fig. 4. The degree of polymerization of CNFs was retained for more than $70 \%$ compared with the raw materials, and there was no change in the XRD pattern.

These results suggest that the water jet treatment breaks intermolecular hydrogen bonds and almost does not breaks the b-1,4-glycosidic bonds in the cellulose molecular chains, but it does not affect the crystal structure; thus, the applied fibrillation is gentle and exerts only the minimum necessary force to produce CNFs. Controlling the degree of polymerization and crystallinity is important because the physical properties of CNFs and CNF-based products vary significantly depending on fiber length and crystallinity. The water jet method allows control of the raw material used, and therefore, of the obtained CNFs to produce CNFs that are suitable for a wide variety of applications.

\subsection{Basic properties of CNF dry powder}

The particle size distributions of the CNF dry powders are shown in Fig. 5. The median diameters of WFoUNDP and FMa-UNDP were 5.9 and $2.5 \mathrm{~mm}$, respectively. The longer the fiber length, the larger is the particle size, because CNFs aggregate loosely and become particulates during the drying process.

\section{Applications Of Cnf Aqueous Dispersions 4.1. Particle dispersant}

\subsubsection{Particle to disperse}

Water-repelling $\mathrm{TiO}_{2}$ (particle size: 0.03-0.05 mm; specific gravity: 4.2), diamond powder (particle size: 4-8 mm; specific gravity: 3.2), and inorganic pigment (particle size: $15 \mathrm{~mm}$ or less; specific gravity: 3.0 ) were used as the particles to be dispersed.

\subsubsection{Dispersion method}

A 2 wt\% CNF aqueous dispersion (BiNFi-s WFo-10002, Sugino Machine Ltd., Toyama, Japan) was treated for $5 \mathrm{~min}$ with a blender (7012S, WARING, USA) at $8400 \mathrm{rpm}$ and then diluted to $0.5 \mathrm{wt} \%$. The particles 
$(0.3 \mathrm{~g})$ were charged into the $0.5 \mathrm{wt} \% \mathrm{CNF}$ dispersion $(15 \mathrm{~g})$. The water-repellent $\mathrm{TiO}_{2}$ was treated using a stirring defoaming machine (Hi-Merger HM-200WV, KYORITSU SEIKI CORPORATION, Tochigi, Japan) at the rotational condition " 9 " for $10 \mathrm{~min}$, while the diamond powder and the inorganic pigment were treated using a propeller stirrer at $500 \mathrm{rpm}$ for $5 \mathrm{~min}$.

\subsubsection{Results and discussion}

Fig. 6 shows the state of the CNF-added and CNF-free particle dispersions after $3 \mathrm{~h}$ and $24 \mathrm{~h}$ of stirring. The CNF-free particle dispersions showed sedimentation and flotation of particles, whereas the CNFadded ones did not show either phenomenon, indicating good dispersibility. Because the particles are retained in the CNF three-dimensional network structure in water, their affinity to water is improved. Therefore, particles characterized by water repellency and high specific gravity can be uniformly dispersed for a long period of time with this method.

\subsection{CNF/Natural rubber (NR) composite}

\subsubsection{Materials}

The CNF aqueous dispersions used for this application were $5 \mathrm{wt} \%$ ultra-long fiber type (BiNFi-s IMa10005, Sugino Machine Ltd., Toyama, Japan) and 5 wt\% ultra-short fiber type (BiNFi-s FMa-10005, Sugino Machine Ltd., Toyama, Japan). NR latex (high ammonia type, S\&S Japan Co., LTD., Hyogo, Japan) were used as NR.

\subsubsection{Compound}

Either CNFs type was added to an NR latex at 5 per hundred rubber (phr) after drying. The mixture was treated using a stirring defoaming machine under rotation condition " 5 " and revolution condition " 9 " for 4 min, and various CNF/NR wet master batches were obtained. The resulting wet master batch was dried at $70{ }^{\circ} \mathrm{C}$ for $48 \mathrm{~h}$ to obtain a CNF/NR master batch. A 5-phr CNF/NR composite was produced by compounding with additive agents (0.5-phr stearic acid, 6.0-phr zinc oxide, 35-phr sulfur and 0.7-phr N-tButyl-2-benzothiazolesulfenamide (as vulcanization accelerator) using a double roll $(f=200 \mathrm{~mm}, L=500$ mm, Mixing Mills, Nippon Roll MFG. Co., Ltd., Tokyo, Japan), molding, and vulcanization using a hot press.

\subsubsection{Tensile strength test}

The 5-phr CNF/NR composites were subjected to a tensile strength test (JIS K 6251) using a precision universal tester (AG-50KNXD, Shimadzu Corporation, Kyoto, Japan). The tensile speed was $500 \mathrm{~mm} / \mathrm{min}$. 


\subsubsection{Results and discussion}

The S-S curves (Fig. 7) showed that the reinforcing effect is different owing to the different fiber lengths of CNF. When the fiber was ultra-long, the initial modulus was greatly improved, and the breaking strain was lowered. On the other hand, when the fiber was ultra-short, the initial modulus was slightly improved, which in turn improved the breaking strain. This is a general phenomenon that occurs when a nanofibrous filler is added NR because CNFs are uniformly dispersed in the rubber. In addition, anisotropy appeared in the tensile strength with the addition of CNF. This is because CNFs were oriented in the same direction as the rotation of the double roll during compounding; thus, the longer the fiber length, the more significant is the difference in tensile strength in the direction of the grain relative to the anti-grain. An interesting result was obtained following the addition of two types of CNFs. The physical properties such as initial modulus and strain of the CNF/NR composites obtained by adding two types of CNFs with different fiber lengths were successfully controlled. In other words, the initial modulus is improved by increasing the ratio of the ultra-long fibers, and the strain can be improved by increasing the ratio of the ultra-short fibers. These results suggest that CNFs can be used to produce composite rubbers that are suitable for various applications and purposes.

\section{Applications Of Cnf Dry Powder 5.1. CNF/polypropylene (PP) composites}

\subsubsection{Materials}

PP was purchased for SunAllomer Ltd. (PX600N, Tokyo, Japan). WFo-UNDP or FMa-UNDP were used as CNF dry powder. Acid modified low-molecular-weight PP (MAPP, UMEX Y-1010, Sanyo Chemical Industries, Ltd., Kyoto, Japan) were used as compatibilizing agent.

\subsubsection{Compound}

CNF dry powder (WFo-UNDP or FMa-UNDP) and acid-modified low-molecular-weight PP were added to PP, and the mixture was compounded using a parallel twin screw lab extruder (Xplore Instruments, Netherland) for 5 min at Melting temperature: $180-200^{\circ} \mathrm{C}$, twin screw speed: $80 \mathrm{ppm}$, to obtain a $1 \mathrm{wt} \%$ CNF/1 wt\% MAPP/PP composite.

\subsubsection{Tensile strength test}

The $1 \mathrm{wt} \% \mathrm{CNF} / 1 \mathrm{wt} \%$ MAPP/PP composites were subjected to a tensile strength test (JIS K 7162) using a precision universal tester. The tensile speed was $10 \mathrm{~mm} / \mathrm{min}$. 


\subsubsection{Results and discussion}

The low-strain side of the resulting S-S curve is shown in Fig. 8(A), and entire curves are shown in Fig. 8(B). The maximum stress in the elastic region and elastic modulus increased approximately 1.1 and 1.3 times, respectively, and no significant improvement was observed with the addition of CNF dry powder. However, the high-strain region is noteworthy. The breaking strain in the case of pure PP was approximately $700 \%$; it was higher than $840 \%$ when the ultra-short fiber type was added, and higher than $970 \%$ when the standard fiber type was added, further confirming that the breaking stress was improved more than the yield stress. Concerning this factor, it is considered that the dispersibility of CNF dry powder in PP was excellent, CNFs acted as a crystal nucleating agent, the PP spherulite size was reduced, and CNFs suppressed the bonding between the smaller PP cracks.

Such phenomena have not been reported before in the examples of applications based on CNFs and they represent unique features.

\section{Conclusion}

The CNF produced by the water jet method is low in contaminations and high in crystallinity, and can be used in various applications. However, there is still a cost challenge.

We have manufactured and sold CNFs in Japan since 2011. We have accumulated various techniques and information over 10 years. From this experience, we recognize that it is important to research properties that can be obtained only by using CNFs. At the same time, we advocate the company philosophy "Glocal (Global + local) Niche Leader" because we conduct global business with niche industries in the local area of Toyama Prefecture. We feel that CNFs are a suitable product for our company's philosophy. We will continue our research to contribute to the development of CNFs.

\section{Declarations}

\section{Acknowledgment}

Development of CNF dry powder was co-research with Prof. Sanada and Prof. Nagata of Toyama Prefectural University, and supported by New Energy and Industrial Technology Development Organization (NEDO, Japan).

\section{Compliance with Ethical Standards}

\section{Disclosure of potential conflicts of interest}

The author declare that I have no conflicts of interest.

\section{Research involving Human Participants and/or Animals}


The author declare that I have no research involving human participants and/or animals

\section{Funding}

Partial financial support was received from New Energy and Industrial Technology Development Organization (NEDO, Japan).

\section{Conflicts of interest/Competing interests}

The author have no conflicts of interest to declare that are relevant to the content of this article.

\section{Availability of data and material}

The author declares data transparency.

\section{Code availability}

Not applicable

\section{Authors' contribution}

An author contributed to the study conception and design. Material preparation, data collection and analysis were performed by Kota Ogura. The final of the manuscript was written by Kota Ogura.

\section{Ethics approval}

Approval

\section{Consent to participate}

Approval

\section{Consent for publication}

Approval

\section{References}

1. Nakagaito A. N. and Yano H. (2004) The effect of morphological changes from pulp fiber towards nano-scale fibrillated cellulose on the mechanical properties of high-strength plant fiber based composites. Applied Physics A, 78(4): 547-552

2. Saito T., Nishiyama Y., Putaux J. L., Vignon M. and Isogai A. (2006) Homogeneous suspensions of individualized microfibrils from TEMPO-catalyzed oxidation of native cellulose. Biomacromolecules, 7: $1687-1691$ 
3. Watanabe Y., Kitamura S., Kawasaki K., Kato T., Uegaki K., Ogura K. and Ishikawa K. (2011) Application of a water jet system to the pretreatment of cellulose. Biopolymers, 95(12): 833-839

4. Chaikaew S., Maeno Y., Visessanguan W., Ogura K., Sugino G., Lee S.H., Ishikawa K. (2012) Application of thermophilic enzymes and water jet system to cassava pulp. Bioresource Technology 126: 87-91

\section{Figures}

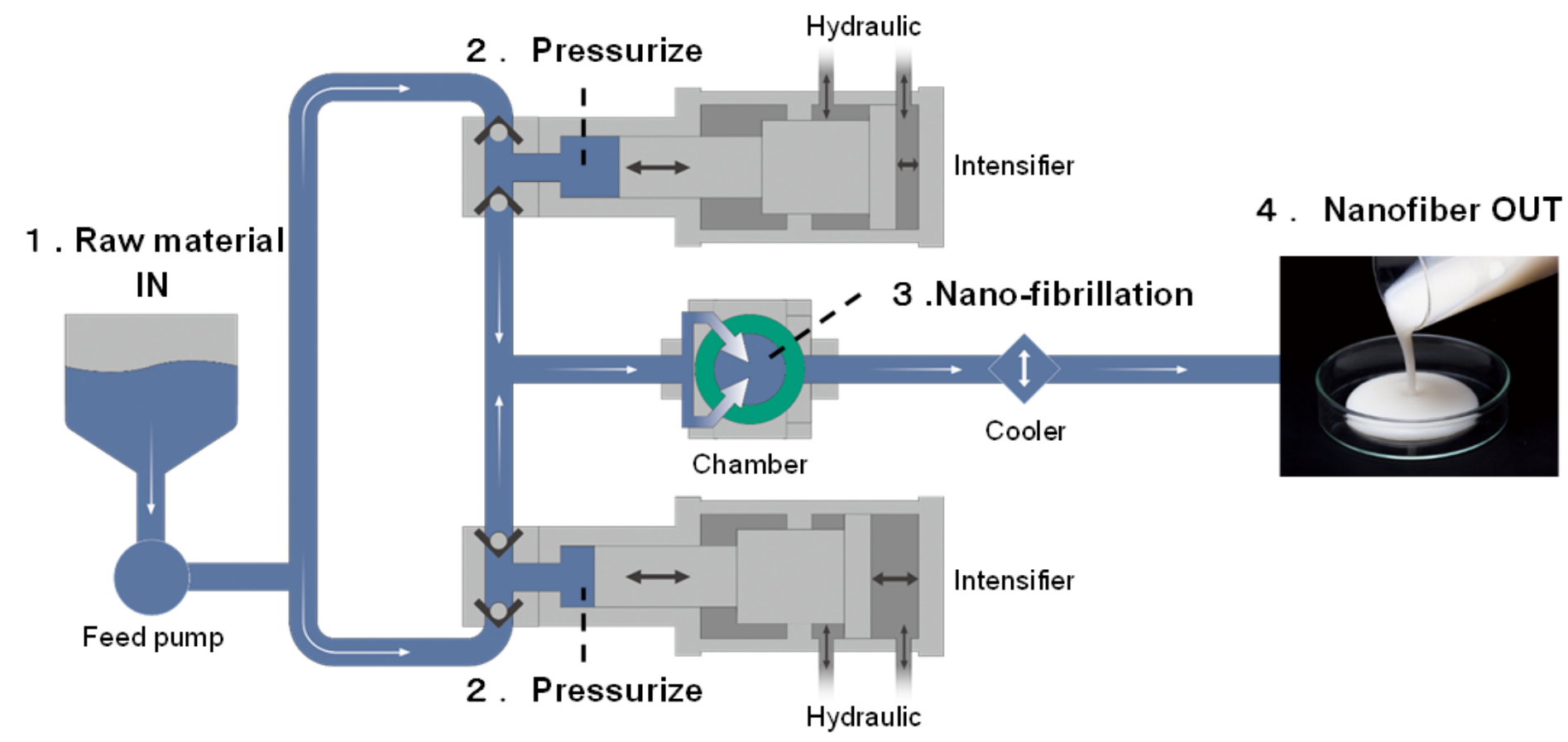

\section{Figure 1}

Equipment used for nanofiber manufacturing via the water jet method 

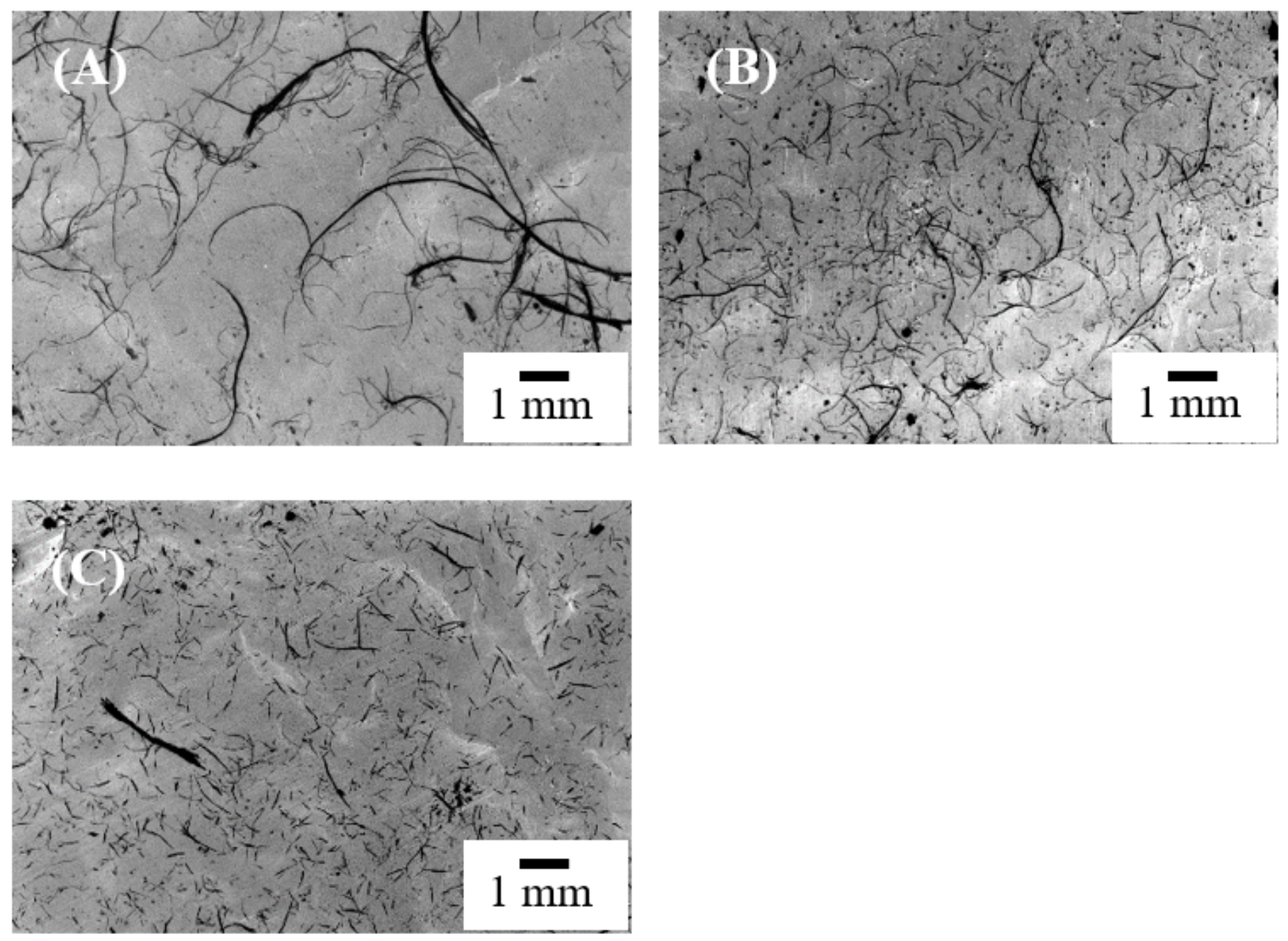

\section{Figure 2}

FE-SEM images of CNFs of various fiber lengths. (A): Ultra long; (B): Standard; (C): Ultra short 


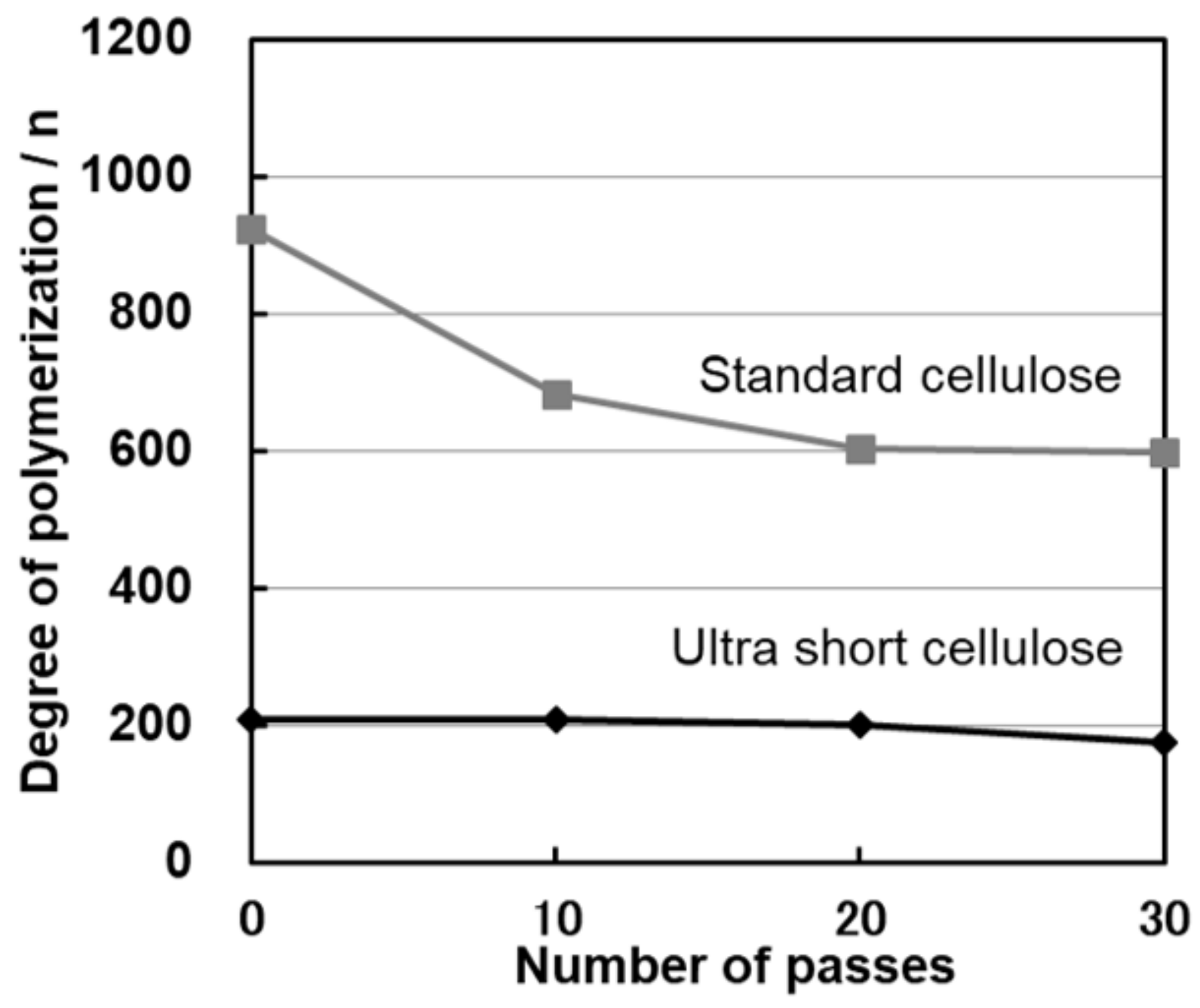

Figure 3

Changes in the degree of polymerization of cellulose after water jet treatment 


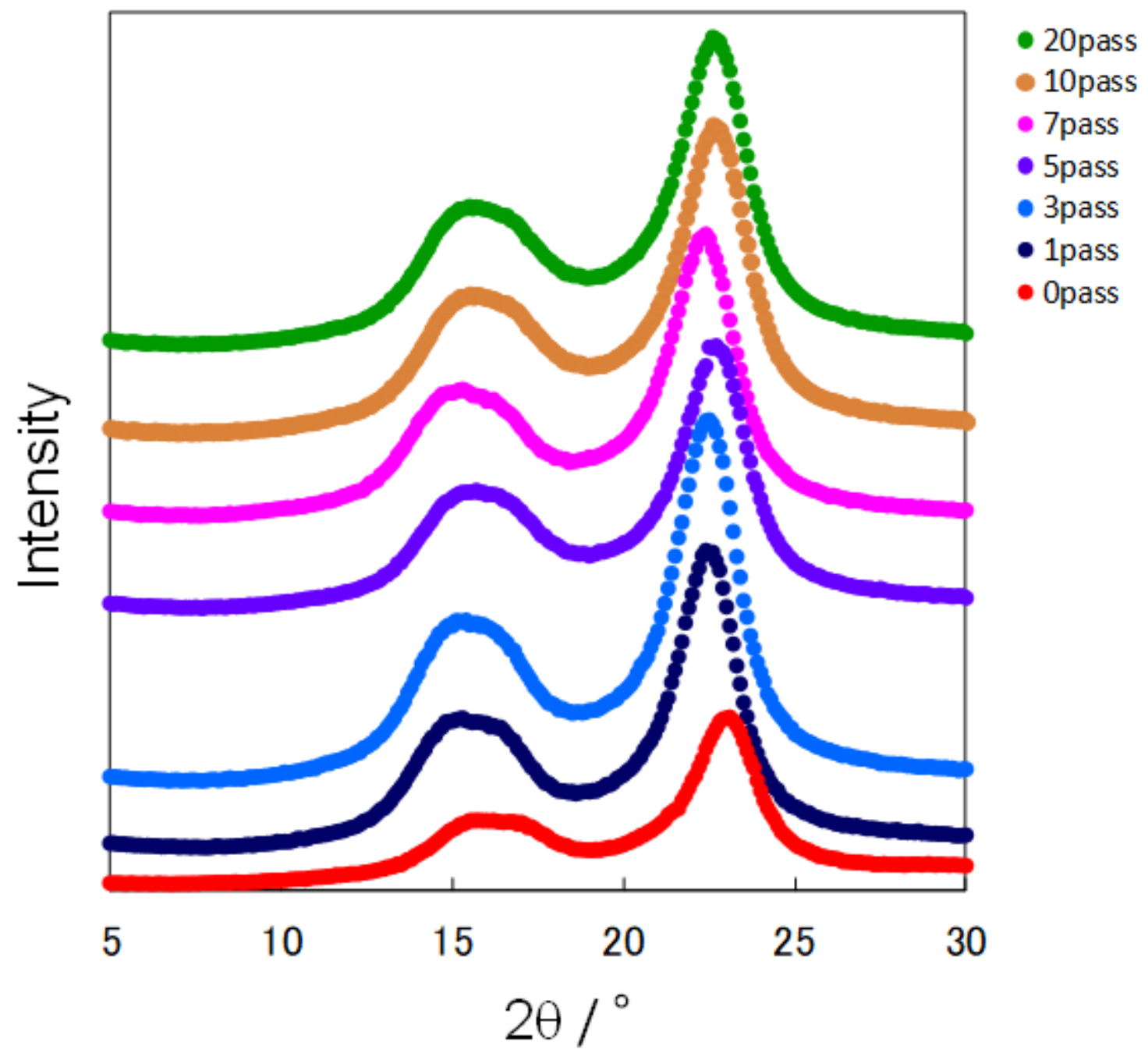

Figure 4

XRD patterns of standard cellulose before and after water jet treatment. (Provided by Dr. Yoshizawa at the National Institute of Advanced Industrial Science and Technology) 


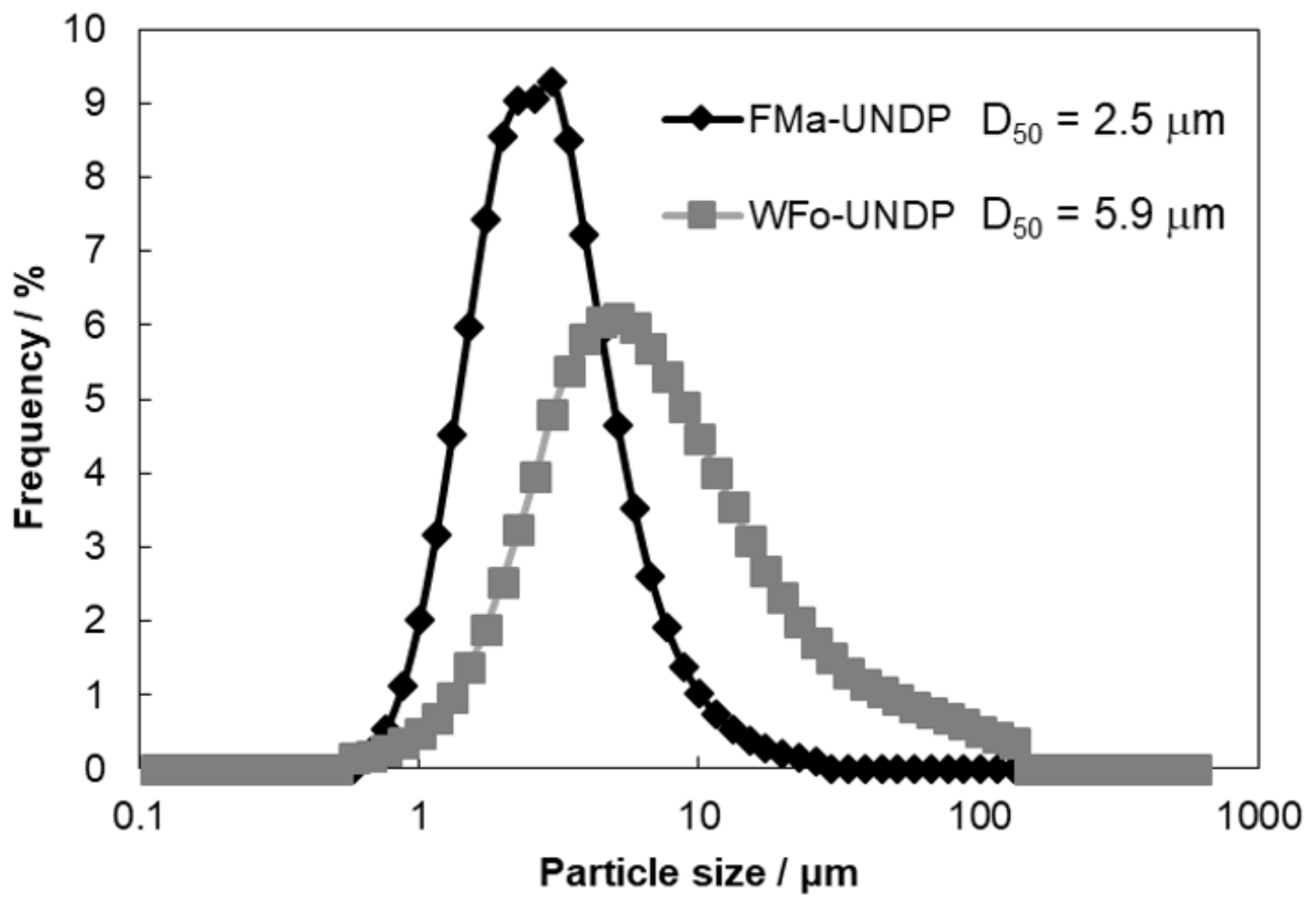

Figure 5

Particle size distribution of CNF dry powder 


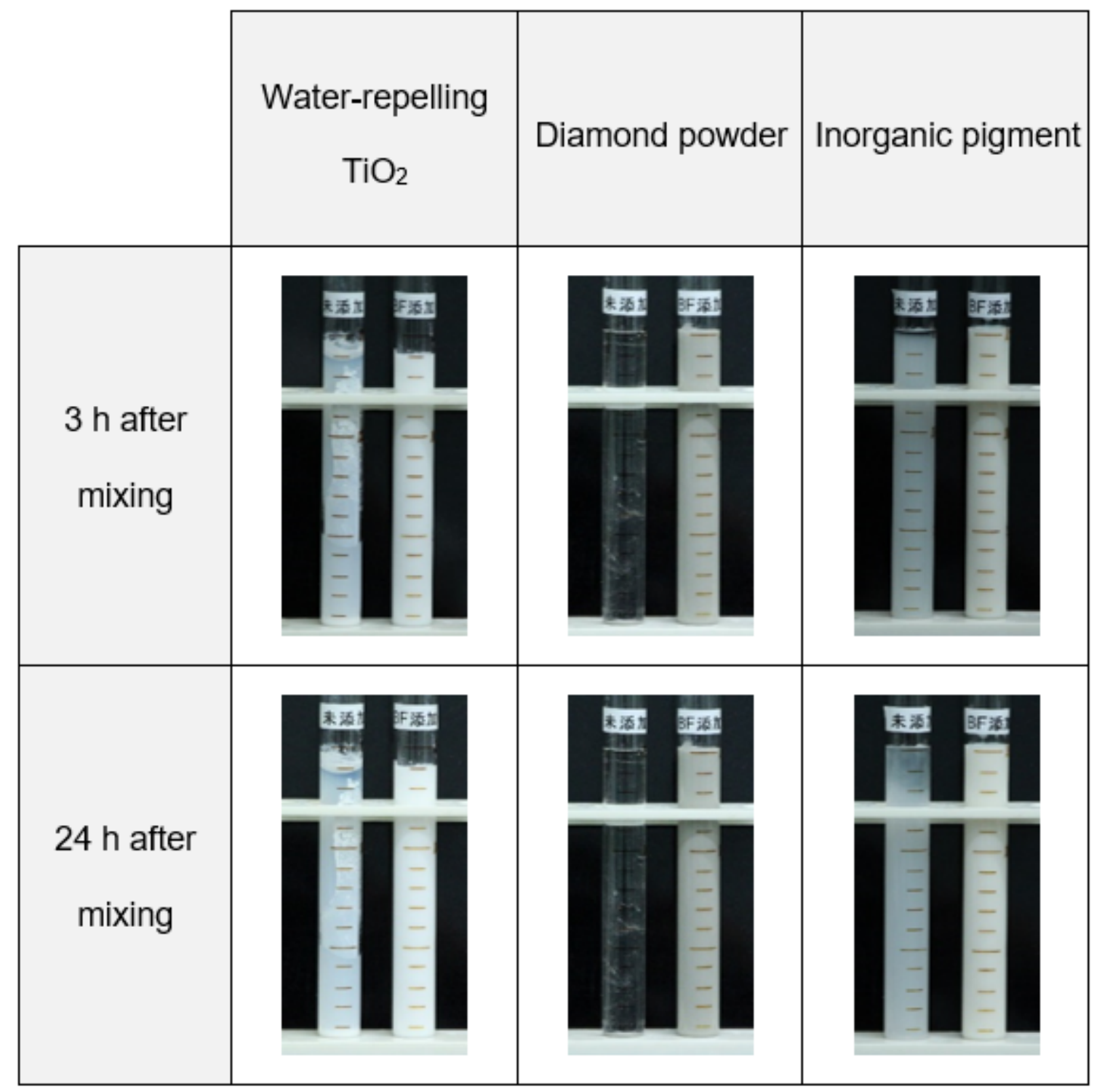

Figure 6

Dispersion stability of various particles after CNF addition. (Right: no CNF; left: CNF addition) 


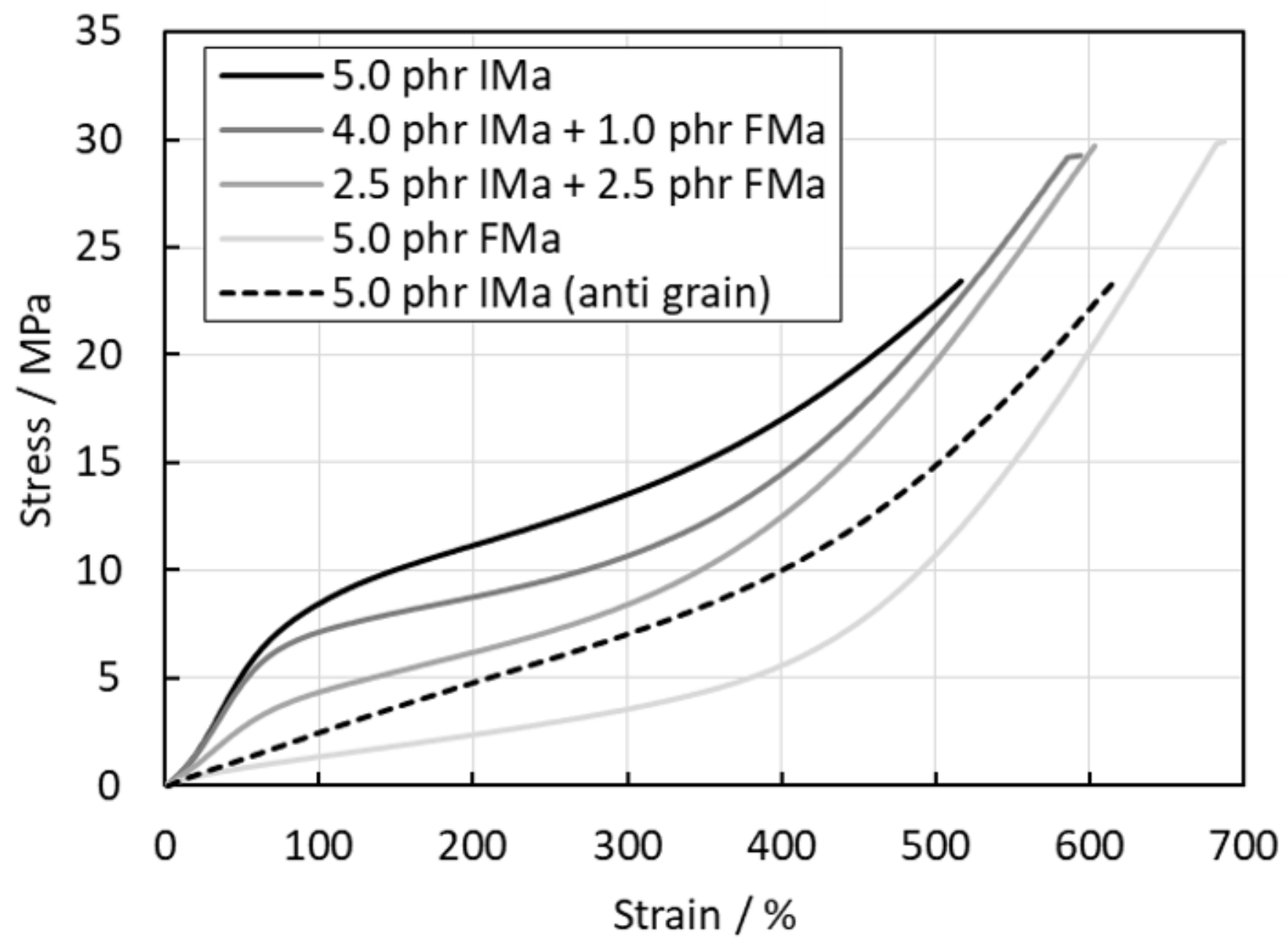

Figure 7

S-S curves of 5-phr CNF/NR composite samples 

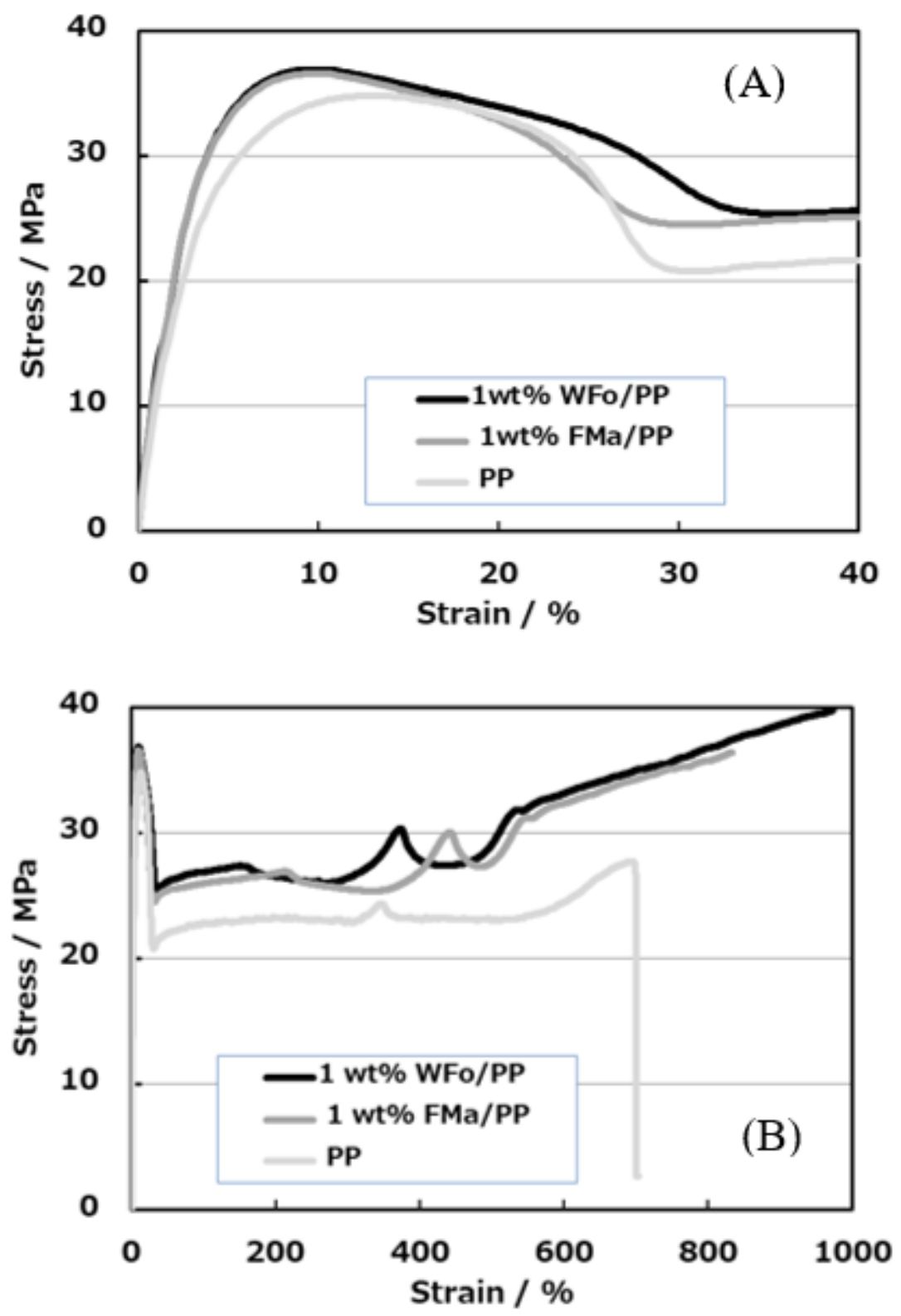

Figure 8

S-S curves of 1 wt\% CNF/PP composite samples. (A) Low strain; (B) All 